\title{
The Domain of the Quantum Matter Wave
}

\author{
B. Mills \\ Institute for Information and Mathematical Sciences, Massey University at Albany, \\ Private bag 102904, NSMC, Auckland, New Zealand \\ Reprint requests to Dr. B. M.; Fax: +64 (0)9441 8181; E-mail: B.I.Mills@massey.ac.nz
}

Z. Naturforsch. 56 a, 216-219 (2001); received January 12, 2001

Presented at the 3rd Workshop on Mysteries, Puzzles and Paradoxes in Quantum Mechanics, Gargnano, Italy, September 17 - 23, 2000.

In quantum mechanics a particle is represented by a complex wave function. This is similar to the classical description of a distributed particle by a real valued density function. However, while classically the combined density of multiple particles can be decomposed into individual densities; the wave function of a multi-particle system can not always be decomposed into wave functions for the individual particles. Hence the classical assumption that density is a property of physical space, which is only possible because of the special topology of classical configuration space, does not work for quantum systems.

Key words: Quantum Matter Wave; Quantum Particle; Quantum Configuration Space.

\section{Induced Topology}

A single particle, whether it is concentrated or distributed, can be modelled by a measure that indicates how much of the particle is in each region of space. In classical mechanics this is a real valued density measure; in quantum mechanics it is complex valued. However, this is a technical detail and does not itself imply a strangeness in quantum mechanics. Some oscillating electro-magnetic fields can be descibed by complex measures, but this does not cause any paradox. The source of quantum entanglement lies elsewhere.

The idea of a configuration space [1] simplifies analysis of the mechanics of multi particle systems. In classical mechanics the configuration space is a direct product of as many copies of physical space as there are particles in the system. In this way two particles in a one dimensional universe become one particle in a two dimensional universe.

Configuration space is often viewed as a useful mathematical construction to be derived from the physical space. However, there is no logical problem in starting with the configuration space, and then the system of particles becomes a useful construction. It becomes a decomposition of the measured configuration space into a direct product of measures on a single underlay- ing space, called physical space. For classical configuration space such a decomposition always exists. The infinitesimal density measure in configuration space is the sum of the individual measures for the particles in physical space:

$$
\mathrm{d} M(a, b)=\mathrm{d} M_{1}(a) \times \mathrm{d} M_{2}(b) .
$$

In quantum theory a corresponding decomposition of a complex valued density measure (a wave function) does not always exist. in order to see why, we will examine the topology of the configuration space of a multi-particle quantum system. When quantum particles cannot be distinguished the configuration space topology is affected. the topology is more complicated because the particles cannot be treated individually. Another viewpoint is that the topology of the configuration space determines when two particles can be distinguished.

What is the topology of an $N$ particle quantum system? We can begin by constructing the classical configuration space consisting of a direct product of $N$ copies of the physical space $X$. A point $\left(x_{1}, \ldots, x_{N}\right)$ in configuration space can be viewed as a tuple whose elements $x_{n}$ are points in physical space corresponding to the position of the individual particles.

But, when, for instance, the first two particles cannot be distinguished, then each point of the form 
$\left(x_{1}, x_{2}, x_{3}, \ldots\right)$ must be identified with its equivalent $\left(x_{2}, x_{1}, x_{3}, \ldots\right)$ in configuration space. This must be done for every pair of particles that cannot be distinguished. The configuration space on which the wavefunction exists is the quotient space $X / \sim$ rather than the physical space $X$.

The quantum density is a measure on this configuration space, not the physical space. When the measured domain is decomposable into measured copies of the physical space $X$, then we can treat the component measures as particles. but, if the space is not thus decomposable, then we are forced to admit that the density measure is defined primary on the configuration space, and it is only sometimes that we can decompose this to obtain measures on the physical space. This strangeness of quantum mechanics is not due to the complex value of the measurement, but to the nature of the domain on which it exists.

\section{Two Particles in Loop Space}

A system of two particles in a one dimensional universe serves as a simple example of the distinction between a classical configuration space, and the corresponding entangled quantum configuration space. The classical configuration space is a torus which can be covered by the plane, thus enabling us to distinguish the two particles. The quantum configuration space is a pseudo sphere which cannot be covered by the plane, showing that the two particles are entangled.

A system of particles in a one dimensional box illustrates the behaviour. The box is taken to be a closed one dimensional universe wrapped around on itself. It can be modelled as a line segment with the end points identified. if ther is only one particle in the box, then the classical and quantum configuration spaces are the same, and isomorphic to the physical space.

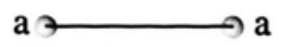

If there are two particles in the box, then the classical configuration space is a direct product of two

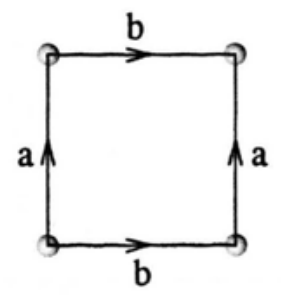

loops. This is a square with opposite edges identified as indicated.

This is a two dimensional manifold with 1 face, 2 edges and 1 vertex, the genus is $1+1-2=0$ and so it is a torus [2].

For the quantum case the configuration space is constructed by starting with the square, but then identifying points in the pattern $\left(x_{1}, x_{2}\right)=\left(x_{2}, x_{1}\right)$.

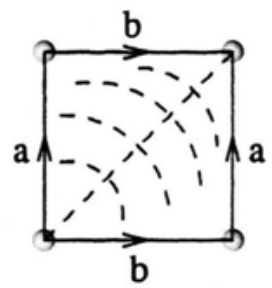

The identification of edges $a=b$ is established directly. The folding also introduces a new edge $c$. Moving across this edge would cause a small rotating disk to be reversed, i. e. $c=c^{-1}$ and so $c=1$.

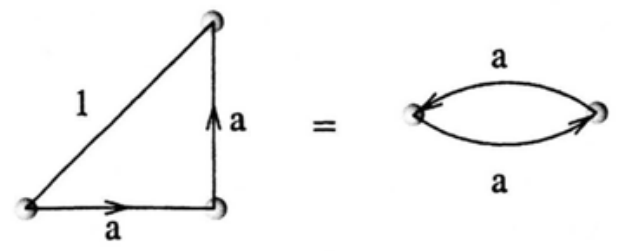

This has 1 face, 1 edge and 1 vertex, $1+1-1=1$, which makes it a pseudo sphere. This means that it is a non-orientable space. A big change from the classical case.

\section{The Quantum Configuration Space}

In classical mechanics the combined density measure for two particles in a one dimensional loop space is defined on a toroidal configuration space which can be decomposed into a direct product of density measures on two copies of physical space. as has been shown, in quantum mechanics the configuration space is a pseudo sphere and cannot be directly decomposed into copies of the physical space. So, the quantum mechanical matter wave is defined on a space which is not directly related to the original space. This space is the space of distinguishable events, the quantum logical event space.

In classical mechanics configuration space is a convenience, a notion, or a point of view, the mass distributions are defined individually for each particle. In quantum mechanics the configuration space is not 
always decomposable into independent copies of the original physical space. Thus the quantum mechanical matter wave is actually defined on the configuration space, not on the physical space. The space of the matter wave may have totally different, much more convoluted, topological properties than the original physical space.

In classical mechanics the density measures can be attached to the particles. This is because the combined measure is a direct product of individual measures. In quantum mechanics, for entangled particles, such a decomposition is not possible, so the density measure is only defined for configurations of the entire system of particles, rather than independently for each particle.

Quantum mechanical wave functiond do not exist in physical space. If we have one particle in the universe we can pretend that the wave function is in physical space because the actual space containing it is homeomorphic to the physical space. However the Quantum mechanical matter wave exists in an event space. For example, if we have two particles in the universe, then we need to determine a complex number for each possible location of both particles at the same time.

The configuration space is a factored direct product of copies of the physical space. This means that it may show topological characteristics that do not exist in the lower dimensions of the physical space; a strong motivation for exploring quantum effects on higher dimensional space.

String theories [3] are constructed using higher dimensional space than perceived physical space because strings in lower dimensional spaces do not behave as required. The quantum configuration space is also a higher dimensional space. If the evolution of quantum systems actually occurs in this configuration space, then the higher dimensions required for string theory are available without them being physical dimensions. Our perceived physical space could then be a projection of a higher dimensional space, rather than being embedded in it.

Are the higher dimensions space not really physical dimensions, but configuration dimensions as above? Can the twisted spaces of string theory be produced as identification spaces in this manner?

\section{Interpretation of the Matter Wave}

Given the natural relation between the quantum configuration space and the probabilistic event space we can try to strictly identify the two. however, probability looses its meaning when the measure of the whole space is infinite. But the concept of frequency retains its meaning. we never really know how many particles are in the universe. but we might know the rate at which they are observed at a certain location.

The occurrence of a particle is like an energy flow. Quantum electro-dynamics is directly related to classical electro-magnetic theory. The rate at which photons are observed at a given location is proportional to the energy density of the field at that time, which is obtained by taking a square magnitude. This gives a strong connection between quantum rates observation, and energy. If we suppose that photons carry the electro-magnetic field, and that they have a given energy associated with them, then the conservation of energy automatically leads to the relation between the frequency of occurrence of photons, and the square of the field strength.

Configuration space and event space are two different names for the same structure. In both cases the quantum mechanical wave function assigns a complex number to each combination of locations of the particles. Neither is a universe of point particles with definite properties, rather the fundamental character is the information obtained from observations.

Viewing the space as an event space we are lead to the idea of conditional event space. Given, for example, that one particle is seen in a particular location, we could construct a configuration space for the rest of the particles. in the case of two electrons in a box the second electon will not occur at the location of the other electron. So the conditional event space is not the same as the configuration space of just one particle. In this way the effective shape of the configuration space, in which the wave function evolves, can be modified by previous observation. Qualitatively, the quantum matter wave is a complex valued density defined on the evolving topology of a high dimensional space of which our observed space is a projection.

\section{Acknowledgements}

The Computer Science Dept, University of Wales, Cardiff, for their generous financial support. Dr. Sonia Schirmer, of the Quantum Process Group, The Open University, UK, for advice and editing assistance. Frank Langbein, University of Cardiff, for 
useful discussion. Auscom Publishing, Sydney Australia, for permission to use the cat picture on the

[1] D. Hestenes, New Foundations for Classical mechanics, Kluwer Academic Press, Amsterdam 1993.

[2] J. Stillwell, Classical topology and combinatorial group theorie, John Stillwell, Springer-Verlag, Berlin 1993. version presented at the conference (omitted in publication for reasons of space).

[3] J. Polchinsky, String Theory, University Press, Cambridge 1998. 